\title{
The role of the speech and language therapist in the assessment and management of dysphagia in neurologically impaired patients
}

\author{
Gillian D. Kennedy
}

Whittington Hospital, Highgate Hill, London N19 5NF, UK

The participation of speech and language therapists in the assessment and management of dysphagic patients is a comparatively recent phenomenon. It is an aspect of their remit with neurologically impaired patients which has gained progressive recognition by many health care workers, ${ }^{1-3}$ whilst others remain sceptical about the appropriateness of the speech and language therapist's involvement with this patient group. Indeed some of the profession's own members consider that this is a disorder in which therapists should not be involved. This view is shared both by those who have little or no experience in the area and by those who are concerned about the considerable drain on already limited resources which is created by providing a service to both patients with dysphagia and those with communicative disability. ${ }^{4,5}$

This latter concern in particular needs to be investigated, but the justification for speech and language therapists undertaking such work is irrefutable. The therapist's work in this area is widely supported by the discipline's own professional bodies. ${ }^{6,7}$ However, the potentially life-threatening nature of the disability, which is very different from other disorders encountered by speech and language therapists, is acknowledged and due care taken to equip therapists with the specialist skills necessary to undertake work effectively with dysphagia patients.

Education on the subject is introduced at undergraduate level but therapists are recommended to attend a recognized training course post-qualification and to subsequently maintain and update their knowledge. ${ }^{8}$ Indeed, many therapists have become experts in this disorder, undertaking further postgraduate studies at both Master and Doctorate level.

Correspondence: G.D. Kennedy, M.Sc., M.C.S.T. Accepted: 17 January 1992
The timing of the speech and langugage therapist's initial involvement with the dysphagic patient is largely dependent on the individual's underlying neurological disorder. For example, a patient presenting with sudden onset of neurological signs may be referred within 24 hours of admission. Alternatively, a patient suffering from a progressive neurological disorder may not be referred until such time as the swallowing problem is having a significant impact on the individual.

All patients with dysphagia should have access to the service via a written medical referral. This ensures that the medical practitioner is in agreement with the swallowing assessment being carried out.

It is interesting to note that the referral form frequently states 'gag reflex absent - evaluate swallow please'. Although an absent or diminished gag reflex has been considered by some authors to contribute to an increased predilection for aspiration, ${ }^{9}$ many clinicians consider that there is little correlation between this feature in isolation and the patient's ability to swallow. This observation would seem to be supported objectively by research findings of Horner et al. ${ }^{10}$ and most recently of Bleach. ${ }^{11}$

Upon receipt of referral, evaluation of the swallow pattern may take one of two forms: objective measurement or subjective clinical procedure. A combination of both measures is considered optimum by many, most notably when a joint assessment is carried out by relevant professionals as detailed by Price et al. ${ }^{12}$

Of the objective measures, videofluoroscopy is the most widely used and its contribution to the assessment process is emphasized by a number of research findings which suggest that silent aspiration cannot be identified using clinical judgement in isolation from this radiological procedure. ${ }^{13,14}$

The benefits of utilizing such a technique seem apparent but consideration should also be given to its limitations. For example, the short controlled sample of the patient's swallow during the X-ray 
may not demonstrate the aspiration which takes place in a normal setting. Similarly the adverse reaction, particularly of elderly patients to the unfamiliar environment and taste of barium can influence their performance and may not be representative of the individual's abilities. ${ }^{15}$

It is also noted by some researchers that videofluoroscopy, whilst usually establishing the presence of aspiration, does not give an indication of the quantity of material aspirated nor the tolerance or ability of the lungs to clear the infiltrate. ${ }^{16}$ These features can only be identified through the use of scintigraphy, ${ }^{17}$ a more sophisticated radiological process than videofluoroscopy, which is, as yet, not widely used with dysphagic patients in this country.

The information obtained from scintigraphy is arguably more useful than solely confirming the presence of aspiration as it may help to identify a potential population of 'safe aspirators'. ${ }^{16}$ These patients can be demonstrated to aspirate prandial contents without adverse respiratory sequelae due to the lungs' tolerance and rapid clearing of the foreign material. For some patients, however, such invasive procedures may be deemed inappropriate and for others there may be no access to these techniques. These facts, plus the limitations noted above, highlight the need for development of a more comprehensive clinical evaluation procedure.

Researchers have recently attempted to meet this requirement and have adopted an increasingly functional approach to evaluating the swallow pattern. ${ }^{18,19}$ Of these evaluations, the forthcoming 'Functional Assessment of Dysphagia' (FAD) perhaps presents as one of the most detailed in-depth procedures furnishing the therapist with considerable information about the patient's swallowing abilities. ${ }^{20}$ It also provides an objective record of responses thus allowing changes in the individual's performance to be monitored more accurately over time.

Such an assessment initially covers areas which influence the patient's swallowing abilities, namely general condition and posture. This information is gathered from observation and advice obtained from other relevant professionals. It progresses to investigation of afferent information, covering structures around the face and oral cavity. Many existing procedures omit this from their repertoire but without appropriate afferent information being received, competent efferent patterns cannot be instigated. After documenting appearance of relevant structures, the patient's motor abilities for swallowing are evaluated on functional activities which emulate the movements of the articulators during the eating and swallowing process. These responses are rated on a five-point scale and enable the therapist to predict where the patient may experience difficulty.

The final section investigates abilities on direct oral intake over the three initial phases of the swallow pattern; namely the oro-preparatory, oral $\frac{3}{3}$ and pharyngeal phases. A prescribed variety of $\stackrel{\varnothing}{\complement}$

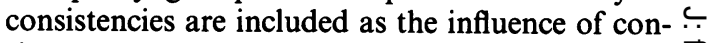
sistency on the eating and swallowing abilities of $\overrightarrow{\vec{D}}$ neurologically impaired patients is well docu- $\frac{T}{0}$ mented. ${ }^{21,22}$ Consequently, the results of this may $\frac{\text { C }}{5}$ indicate that modification of diet alone would $\frac{\bar{\sigma}}{\bar{\omega}}$ minimize risk of aspiration thus negating the need $\vec{\Phi}$ for alternative regimens to be considered.

On oral intake, seven significant areas are eval- $\stackrel{\infty}{\infty}$ uated: (1) lip closure on retrieval from vessel; (2) lip $\overrightarrow{0}$ closure throughout oral phases; (3) lateral tongue $\overrightarrow{-}$ movement during mastication (on appropriate $\vec{\sigma}^{-}$ consistencies); (4) tongue movement on posterior $\frac{}{0}$ propulsion of bolus; (5) triggering of the swallow $\frac{0}{3}$ reflex. Here the excursion and timing of laryngeal movement is measured by both visual and tactile $\infty$ monitoring to allow for more accurate evaluation ${ }^{\circ}$ of the competence and effectiveness of the swallow ir pattern. Such measurement is suggested but not $f$ stipulated, in only one other published assess- 0

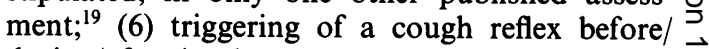
during/after intake on each consistency; and (7) $\vec{c}$ voice quality following intake on each consistency. Once again items are rated on a five-point scale to $\vec{\varphi}$ reflect change on reassessment.

Comparison is warranted between this type of procedure, which is widely used by speech and language therapists, and that employed in medicat studies to determine the incidence of dysphagia in neurologically impaired patients. An example of the latter may be taken from the study of Gordon and colleagues ${ }^{23}$ who aimed to establish the incidence of dysphagia in stroke patients admitted to an acute general hospital. Here dysphagia was evaluated by observing the patient's ability to drink $50 \mathrm{ml}$ of water. Dysphagia was considered to be present if the patient coughed during attempts to drink. Whilst coughing or choking on intake is a sure sign of aspiration, the study does not account for the number of patients who may have been silent aspirators. Moreover, it is suggested that this form of assessment, whilst arguably being adequate for audit purposes, does not have application to day-to-day patient management as the subsequent information gained does not enable the team to make reliable judgements concerning the patient's suitability or otherwise for continued oral intake. Conversely, the results of a comprehensive clinical c assessment as described earlier enable the speech $\underset{2}{ }$ and language therapist to establish the patient's potential for aspiration and to provide this information to relevant disciplines.

It is proposed that the subsequent decision regarding management of the patient should not be the responsibility of one professional health care group alone. Instead, the multidisciplinary team should reach a joint decision regarding future 
management based on such factors as assessment findings, medical condition, and not least, the wishes of the patient and/or family. The need for joint decision is again reinforced by acknowledgement of the fact that the subject of feeding is an emotive issue. This is well illustrated by several researchers ${ }^{24}$ and further emphasized by the wealth of debate created by the recent ethical cases in the USA involving proposed withdrawal of alternative feeding regimens from patients who are in a persistent vegetative state. ${ }^{25-27}$ If decisions regarding proposed management of the dysphagic patient are not made in conjunction with all relevant personnel, it is likely that professional groups may become antagonistic and estranged, ${ }^{28}$ neither of which is conducive to good patient care.

The speech and language therapist's subsequent intervention following assessment is largely determined by the outcome of the multidisciplinary team's discussions. However, other factors also influence the type of treatment offered. A recent preliminary study suggests that these other aspects include motivation, cognitive function and availability of support. ${ }^{29}$

Contingent upon the above, the management undertaken will either involve active therapy or advice only to patient and/or carer. Active therapy is directed towards improving the swallow pattern and minimizing the potential for aspiration. Specific exercises are given to improve oromotor and laryngeal function during the eating and swallowing process. These may include thermal stimulation or teaching compensatory strategies such as a supraglottic swallow or head rotation. The value of such applied treatment techniques to the recovery process of the dysphagic patient is well observed clinically and is supported by research findings. ${ }^{30-32}$

The aim of providing advice alone without employing specific treatment techniques is also to

\section{References}

1. Wiles, C.M. Neurogenic dysphagia. J Neurol Neurosurg Psychiat 1991, 54: 1037-1039.

2. Drinka, P., Schmidt, G. \& Voeks, S. Brain damaged patients with swallowing disorders (Letter). J Am Geriatr Soc 1991, 39: 315-319.

3. Mutch, W.J., Strudwick, A., Roy, S.K. \& Downie, A.W. Parkinson's disease: disability review and management. $\mathrm{Br}$ Med J 1986, 293: 675-677.

4. O'Leary, I. Personal viewpoint: who should be working on dysphagia? Bull Coll Speech Lang Therap 1991, 470: 7.

5. Edelman, G. Dysphagia series: increased clinical activity raises major questions. Speech Therapy Pract 1989, February, 13-14.

6. College of Speech and Language Therapists. Communicating Quality. The Collge of Speech and Language Therapists, London. 1991, pp. 182-187. minimize risk of aspiration on oral intake and to increase patient comfort. However, this is achieved through more indirect methods with patients for whom active, more aggressive therapy is considered inappropriate. Such patients may include those in the final stages of a progressive illness, those with significant cognitive deficits or the very elderly. ${ }^{15}$

Here the patient and/or carer may be advised on certain aspects including positioning, modification of dietary consistencies and optimum environmental conditions to encourage and facilitate a safer, more pleasurable feeding situation. Such advice is often given in collaboration with connected agencies, notably physiotherapy, dietetics and occupational therapy.

Irrespective of the proposed type of intervention, medical agreement should be sought prior to instigation and related disciplines informed of this and any future change in approach. This liaison between relevant professions is necessary to confirm that safe feeding practices are implemented thus ensuring the patient's well-being.

In conclusion, the role of the speech and language therapist in the assessment and management of dysphagic patients is not to be the primary decision maker. Instead the speech and language therapist has a responsibility to use his/her specialist skills to evaluate the swallowing process thoroughly, provide the multidisciplinary team with the results of such procedures, participate in the decision-making process regarding management, and implement appropriate intervention strategies as agreed by the medical team. In this way, the speech and language therapist may contribute greatly to patient care, and reduce or limit the need for costly resources being required for neurological patients where aspiration on oral intake may be a likely consequence of dysphagia.

7. American Speech-Hearing Association Ad Hoc Committee on Dysphagia. Skills needed by speech-language pathologists providing services to dysphagic patients/clients. American Speech and Language Association 1990, 32: 7-12.

8. College of Speech and Language Therapists Dysphagia Working Party. Position Paper on Dysphagia. College of Speech and Language Therapists, London, 1990.

9. Linden, P. \& Siebens, A.A. Dysphagia - predicting laryngeal penetration. Arch Phys Med Rehabil 1983, 64: 281-284.

10. Horner, J., Massey, E.W., Riski, J.E., Lathrop, D.L. \& Chase, K.N. Aspiration following stroke: clinical correlates and outcome. Neurology 1988, 38: 1359-1362. 
11. Bleach, N.R. The gag reflex and aspiration: a retrospective study of 120 neurology patients assessed by videofluoroscopy. Lecture given December 1991 at Dysphagia Meeting, Charing Cross Hospital, London. (Paper to be submitted for publication.)

12. Price, G.J., Jones, C.J., Charleton, R.A. \& Allen, C.M.C. A combined approach to the assessment of neurological dysphagia. Clin Otolaryngol 1987, 12: 197-201.

13. Horner, J. \& Massey, E.W. Silent aspiration following stroke. Neurology 1988, 38: 317-319.

14. Splaingard, M.L., Hutchins, B., Sulton, L.D. \& Chaudhuri, G. Aspiration in rehabilitation patients - videofluoroscopy vs. bedside clinical assessment. Arch Phys Med Rehabil 1988, 69: $637-640$.

15. Feinberg, M.J., Knebl, J., Tully, J. \& Segall, L. Aspiration and the elderly. Dysphagia 1990, 5: 61-71.

16. Silver, K., Van Nostrand, D., Kuhlemeier, K. \& Siebens, A. The use of scintigraphy to detect and semiquantify subglottic aspiration (Abstract). Dysphagia 1990, 5: 111

17. Muz, J., Mathog, R.H., Miller, P.R., Rosen, R. \& Borrero, G. Detection and quantification of laryngotracheopulmonary aspiration with scintigraphy. Laryngoscope 1987, 97: 11801185.

18. Cherney, L.R., Cantieri, C.A. \& Pannell, J.J. RIC Clinical Evaluation of Dysphagia. Aspen Systems Corporation, Maryland, 1986.

19. Warms, T., Champion, R. \& Mortensen, L. The Parramatta Hospital's Assessment of Dysphagia. Westmead, New South Wales, 1991.

20. Kennedy, G.D. A functional assessment of dysphagia. Speech Therapy Pract 1991, April, 27-28.

21. Logemann, J.A. Evaluation and Treatment of Swallowing Disorders. College Hill Press, San Diego, 1983.
22. Groher, M.E. (ed.) Dysphagia: Diagnosis and Management. Boston, Butterworth, 1984

23. Gordon, C., Langton Hewer, R. \& Wade, D.T. Dysphagia in acute stroke. $\mathrm{Br}$ Med J 1987, 295: 411-414.

24. American Nurses Association. Withdrawing or withholding $\subseteq$ food and fluid - excerpts from guidelines from the ANA $\Rightarrow$ Committee on Ethics. Am J Nursing 1988, 88: 797-801.

25. Bopp. J. Reconciling autonomy and the value of life. J Am Geriat Soc 1990. 38: 600-602.

26. Glover. J.J. The case of Ms Nancy Cruzan and the care of the elderly. J Am Geriat Soc 1990. 38: 588-593.

27. Pearlman, R.A. Clinical fallout from the Supreme Court Decision on Nancy Cruzan: Chernobyl or Three Mile Island?

28. Watts. D.T., McCaulley, B.L. \& Priefer, B.A. Physician - $\vec{\circ}$ nurse conflict: lessons from a clinical experience. J Am Geriat Soc 1990. 38: $1151-1152$.

29. Walshe, M.M. Management decisions in adult dysphagia: a $\underset{\omega}{\omega}$ study of the factors influencing patient selection and recommended therapeutic intervention. Unpublished M.Sc. Thesis City University, London, 1991.

30. Lazzara, G., Lazaru, C. \& Logemann, J.A. Impact of thermal stimulation on the triggering of the swallow reflex. Dysphagia 1986. 1: 73-77.

31. Logemann. J.A.. Kahilas, P.J., Kabara, M.\& Vakil, V.B. The benefit of head rotation on pharyngoesophageal dysphagia. Arch Phis Med Rehabil 1989, 70: 767-771.

32. Logemann, J.A. Relearning to swallow after stroke - application of manoeurres and indirect biofeedback: a case study. Neurology 1990, 40: 1136-1138. 